\title{
A GENERALIZED METHOD FOR DERIVING MASS-LOSS \\ RATES:

\author{
THE FIRST ORDER MOMENT OF UNSATURATED \\ P CYGNI LINE PROFILES
}

\author{
J. SURDEJ* \\ Institut d'Astrophysique, Université de Liège, Belgium
}

(Received 3 September, 1982)

\begin{abstract}
For the case of optically thin lines, we show that the relation existing between the first order moment $W_{1} \alpha \int\left(E(\lambda) / E_{c}-1\right)\left(\lambda-\lambda_{12}\right) \mathrm{d} \lambda$ of a P Cygni profile and the quantity $\dot{M}$ (level), where $\dot{M}$ is the mass-loss rate of the central star and $n$ (level) the fractional abundance of the ion under study, is in fact independent of any Sobolev-type approximations used for the transfer of line radiation, contrary to what has been assumed in some previous works (Castor et al., 1981; Surdej, 1982). Consequently, all results established in the context of "very rapidly' expanding atmospheres and mainly referring to the non-dependence of $W_{1}$ vs various physical (underlying photospheric absorption line, limb darkening, etc.) and geometrical (velocity field $v(r)$, etc.) effects remain unchanged for arbitrary (e.g. non-Sobolev type) outward-accelerating velocity laws.

Whenever applied with caution, Equation (50) thus provides a very powerful means of deriving mass-loss rates - with a total uncertainty less than 60 percent - from the measurement $W_{1}$ of unsaturated P Cygni profiles observed in the spectrum of early - as well as late - type stars, quasars, etc.
\end{abstract}

\section{Introduction}

In the framework of the Sobolev approximation (Sobolev, 1947, 1957, 1958; Castor, 1970; Lucy, 1971), Castor et al. (1981, referred to below as CLS) have established for the case of optically thin lines that the first order moment $W_{1} \alpha \int\left(E(\lambda) / E_{c}-1\right)\left(\lambda-\lambda_{12}\right) \mathrm{d} \lambda$ of a $\mathrm{P}$ Cygni line profile is proportional to the quantity $\dot{M} n$ (level), where $\dot{M}$ is the mass-loss rate of the central star and $n$ (level) the fractional abundance of an ion in the lower atomic level associated with the given line transition $1 \rightleftharpoons 2$. Whereas CLS have applied this relation for specific distributions of the velocity field $\left(X^{\prime}=1-1 / L\right)$ and of the fictive radial opacity $\left(\tau_{12}^{r}\left(X^{\prime}\right) \alpha\left(1-X^{\prime}\right)\right)$, Surdej (1982, hereafter referred to as Paper I) has shown that under the reasonable assumption of mass-conservation of the relevant species in the flow, the relation $W_{1} \alpha \dot{M} n$ (level) was in fact nearly independent on various physical and geometrical effects such as the type of 'supersonic' velocity law $v(r)$, collisions $(\varepsilon \neq 0)$ and/or the superposition of a rotational velocity field $v^{\perp}(r)$ in the atmosphere, the presence of an underlying photospheric absorption line, an unresolved resonance doublet profile, a (strong) limb darkening law $\psi\left(\mu^{*}\right)$ for the central core, etc. For more details about these results and conclusions, we refer the reader to Paper I.

* Chercheur Qualifié au Fonds National de la Recherche Scientifique, Belgium. 
In the present work, we demonstrate that the relation existing between the first order moment $W_{1}$ and $\dot{M} n$ (level) equally applies for arbitrary (e.g. non-Sobolev type) outwardaccelerating envelopes from which $\mathbf{P}$ Cygni type profiles are observed. Therefore, it is concluded that the first order moment $W_{1}$ of an unsaturated $\mathrm{P}$ Cygni line profile provides a powerful means of deriving mass-loss rates from early- as well as late-type stars, quasars, etc. whenever a good estimate for $n$ (level) is available.

\section{General Hypotheses}

In the following, we consider spherically symmetric envelopes in expansion with a radial velocity $v(r)$ around a central stellar core with radius $R^{* \dagger}$. We assume that the flowing atoms are scattering radiation in a resonance line transition $1 \rightleftharpoons 2$ and that, in the frame of the moving atoms, the emitted photons are completely redistributed in frequency and direction. At a distance

$$
L=r / R^{*} \text {, }
$$

let $\Phi_{L}\left(v-v_{12}\right)$ (see Figure 1) represent an arbitrary - but symmetric - function describing the interaction of the two levels ( 1 denoting the lower and 2 the upper one) with photons around the central frequency $v_{12}$ of the transition. As usually, we suppose that $\Phi_{L}\left(v-v_{12}\right)$ is normalized to unity when integrated over frequency and equal to zero outside the interval $\left[v_{12}-\Delta v / 2, v_{12}+\Delta v / 2\right], \Delta v(L)$ being the maximum width of the absorption profile due to turbulent, chaotic, etc. motions of the atoms.

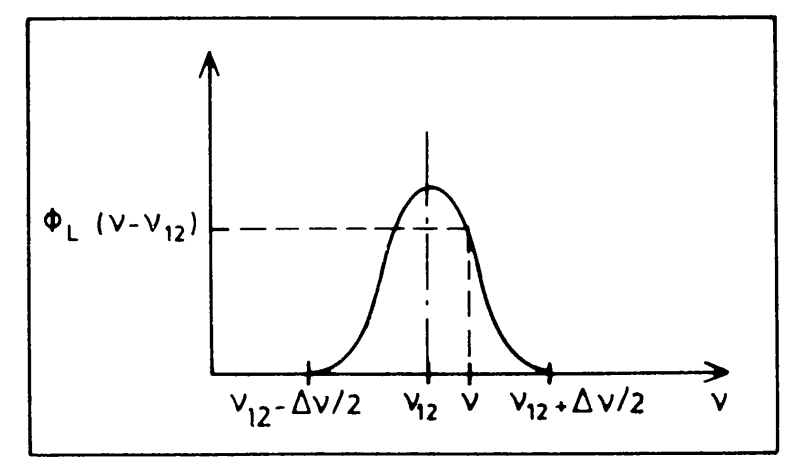

Fig. 1. Absorption profile function in the frame of the moving atoms (see text).

In terms of the dimensionless frequency

$$
X=-\frac{\left(v-v_{12}\right)}{\left(v_{\max }-v_{12}\right)}
$$

where, in accordance with the Doppler relation

$$
\frac{v-v_{12}}{v_{12}}=\frac{v(L)}{c} \mu,
$$

\footnotetext{
+ We stress that this situation could refer to a very slowly expanding atmosphere around a late-type star.
} 


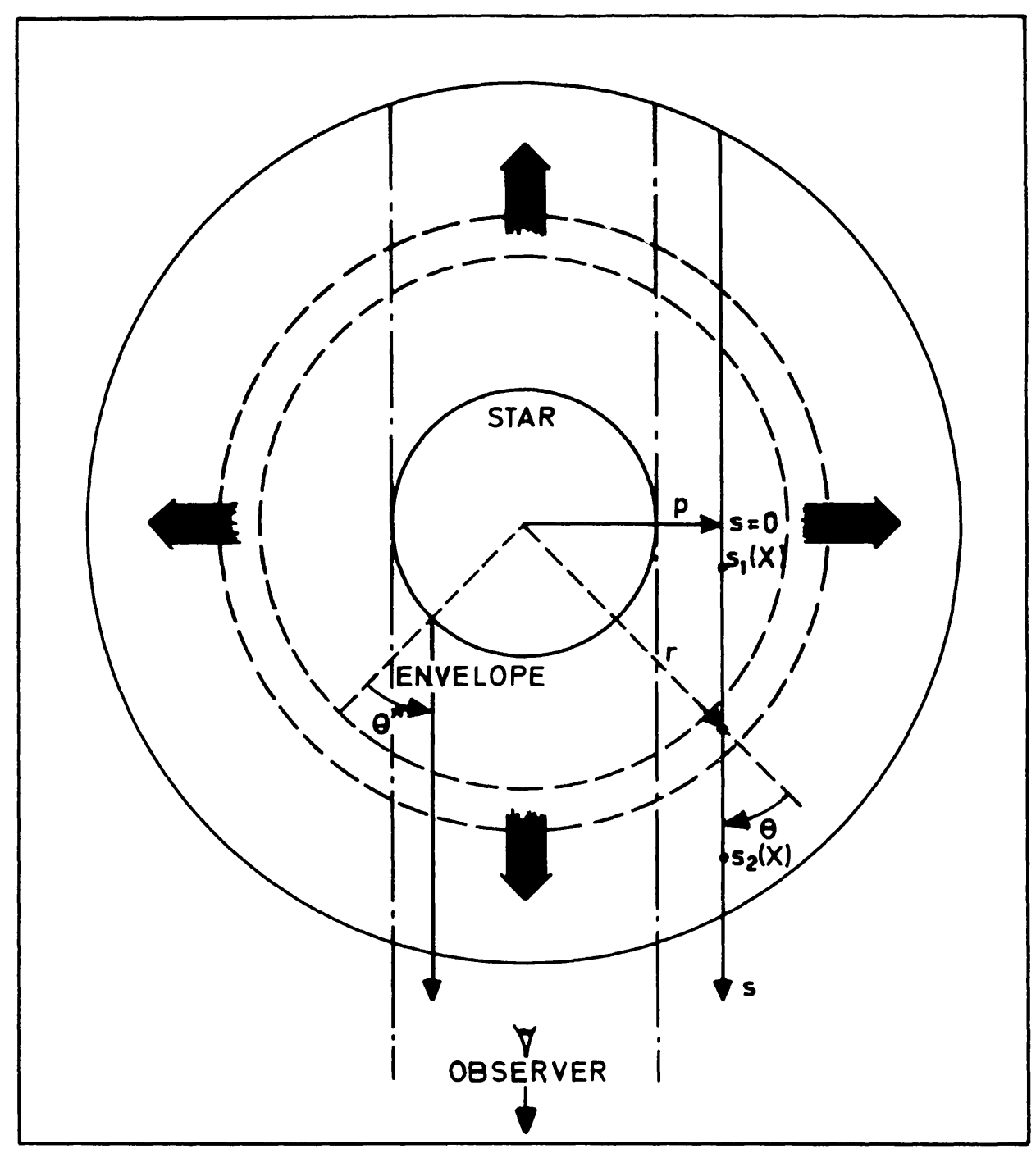

Fig. 2. Geometry in an expanding envelope around a stellar core (see text). No scale is respected.

in which $v$ (resp. $v_{\max }$ ) is the frequency at which a fixed observer receives radiation scattered in the envelope at a point $L$ (resp. $L_{\text {max }}$ ) along a direction $\mu=\cos (\theta)$ (resp. $\mu=1$; see Figure 2), the expression of the line opacity $\tau_{12}^{p}(X)$ evaluated at a frequency $X$ along a direction parallel to the line of sight with abscissae $s$ and impact parameter $p$ is given by

$$
\tau_{12}^{p}(X)=\int_{s p(X)}^{s p(X)} n_{1}(L) k_{12} \Phi_{L}\left(v_{L}-v_{12}\right) \mathrm{d} s .
$$

In this equation, the local frequency $v_{L}$ defined by

$$
v_{L}=v-v_{12} \frac{v(L) \mu}{c}
$$


refers to the frequency of a line photon that would be seen by an observer moving with the medium at $L$ along the direction $\mu$.

With

$$
X_{L}=\frac{-v(L) \mu}{v_{\max }}
$$

representing the dimensionless local frequency at which a stellar photon would be scattered in the line center by atoms moving with a velocity $v(L) \mu$, we easily establish by means of Equations (2), (3), and (5) the useful relation

$$
X_{L}=X+\frac{v_{L}-v_{12}}{v_{\max }-v_{12}} .
$$

For the case of outward-accelerating envelopes, the coordinates $\left(L_{1}, \mu_{1}\right)\left(\operatorname{resp} .\left(L_{2}, \mu_{2}\right)\right)$ of the integration limit $s_{1}^{P}(X)$ (resp. $s_{2}^{p}(X)$ ) appearing in Equation (4) are directly obtained by posing $v_{L}=v_{12}+\Delta v / 2$ (resp. $v_{L}=v_{12}-\Delta v / 2$ ) in relation (7). Of course, this necessarily implies the dependence of $\Delta v$ to be known as a function of $L$. Finally, let us mention that the quantity $n_{1}(L)$ in Equation (4) represents the volume population of the ground level and that - neglecting stimulated emission - the absorption coefficient $k_{12}$ is expressed by

$$
k_{12}=\frac{\pi e^{2}}{m c} f_{12},
$$

where $\pi e^{2} / m c$ is the classical cross-section and $f_{12}$ the oscillator strength of the line transition.

\section{Expression of an Unsaturated P Cygni Line Profile}

Given the values $X_{L}= \pm 1$ and $v_{L}=v_{12} \mp \Delta v / 2$ in relation (7), we directly find that in the rest frame of a fixed observer a $P$ Cygni profile is formed within the frequency interval $X \in\left[X_{\min }^{(1)}, X_{\max }^{(2)}\right]$, where

and

$$
\left.\begin{array}{l}
X_{\min }^{(1)}=-1-\frac{\Delta v / 2}{v_{\max }-v_{12}}, \\
X_{\max }^{(2)}=1+\frac{\Delta v / 2}{v_{\max }-v_{12}},
\end{array}\right\}
$$

$\Delta v / 2$ being evaluated at the maximum radius $L_{\max }$ of the expanding envelope.

Let $E(X)$ be the total amount of spectral energy, defined per frequency and solid angle units, radiated by the medium towards a fixed observer at a frequency $X \in\left[X_{\min }^{(1)}, X_{\max }^{(2)}\right]$. The quantity $E(X)$ is then simply given by the integration of the monochromatic function $I^{p}(X)$ over a plane perpendicular to the line of sight. In the frame of a fixed observer 
$I^{p}(X)$ accounts for the intensity of line radiation emitted along a direction parallel to the line of sight for the given values of the frequency $X$ and impact parameter $p$. Due to the symmetry of the envelope (see Figure 2), we have

$$
E(X)=2 \pi \int_{0}^{r_{\max }} I^{p}(X) p \mathrm{~d} p .
$$

Making the clear distinction between the contributions due to unscattered $\left(I_{u}^{p}(X)\right)$ and scattered $\left(I_{s}^{p}(X)\right)$ stellar radiation to the monochromatic intensity $I^{p}(X)$, we easily find that

$$
I_{u}^{p}(X)=I_{c} \psi\left(\mu^{*}\right) \exp \left(-\tau_{12}^{p}(X)\right), \quad \text { if } \quad p \in\left[0, R^{*}\right] ;
$$

in which we have assumed that the specific intensity radiated by the central source along a direction $\mu^{*} \in[0,1]$ is

$$
I_{c}\left(\mu^{*}\right)=I_{c} \psi\left(\mu^{*}\right)
$$

$\psi\left(\mu^{*}\right)$ being the limb darkening law along the direction $\mu^{*}=\cos \left(\theta^{*}\right)$ (see Figure 2$)$ and that

$$
\begin{aligned}
I_{s}^{p}(X)= & \int_{s_{1}^{\prime}(X)}^{s p(X)} \varepsilon_{12}(s) \Phi_{L}\left(v_{L}-v_{12}\right) \exp \left(-\int_{s}^{s ?(X)} n_{1}\left(L^{\prime}\right) k_{12} \Phi_{L^{\prime}}\left(v_{L^{\prime}}-v_{12}\right) \mathrm{d} s^{\prime}\right) \mathrm{d} s \\
& \text { if } \quad p \in\left[0, r_{\max }\right]
\end{aligned}
$$

with $\varepsilon_{12}(s)$ denoting the volume emission coefficient calculated in a frame moving with the fluid.

With the definition of the integrated stellar flux

where

$$
E_{c}=2 \pi R^{* 2} \int_{0}^{1} I_{c} \psi\left(\mu^{*}\right) L_{p} \mathrm{~d} L_{p},
$$

$$
L_{p}=p / R^{*}
$$

and

$$
\mu^{*}=\sqrt{1-L_{p}^{2}}
$$

the expression of the line profile function $E(X) / E_{c}$ may be written as

$$
\frac{E(X)}{E_{c}}=\frac{2 \int_{0}^{L_{\max }}\left(I_{u}^{p}(X)+I_{s}^{p}(X)\right) L p \mathrm{~d} L_{p}}{\bar{I}_{c}}
$$


where

$$
\bar{I}_{c}=2 I_{c} \int_{0}^{1} \psi\left(\mu^{*}\right) \mu^{*} \mathrm{~d} \mu^{*}
$$

represents the mean specific intensity of the stellar continuum.

For the case of optically thin lines - i.e., assuming $\exp \left(-\tau_{12}\right) \sim 1-\tau_{12}-$ and taking into account the occultation effect for line radiation emitted with positive frequencies towards a fixed observer, the P Cygni line profile function reduces to

$$
\frac{E(X)}{E_{c}}=\frac{E_{u}(X)}{E_{c}}+\frac{E_{s}(X)}{E_{c}}
$$

with

$$
\frac{E_{s}(X)}{E_{c}}=\frac{E_{s}^{(1)}(X)}{E_{c}}+\frac{E_{s}^{(2)}(X)}{E_{c}}
$$

where

$$
\frac{E_{u}(X)}{E_{c}}=1-\frac{2 I_{c}}{\bar{I}_{c}} \int_{\operatorname{Max}\left(0, \mu_{1}^{*}\right)}^{\operatorname{Inf}\left(1, \mu_{2}^{*}\right)} \psi\left(\mu^{*}\right) \tau_{12}^{p}(X) \mu^{*} \mathrm{~d} \mu^{*},
$$

and

if

$$
\begin{aligned}
& \frac{E_{s}^{(1)}(X)}{E_{c}}=\frac{2}{\bar{I}_{c}} \int_{\operatorname{Max}\left(0, \mu_{1}^{*}\right)}^{\operatorname{Inf}\left(1, \mu_{2}^{*}\right)} \int_{s_{1}^{P}(X)}^{s \frac{P}{2}(X)} \varepsilon_{12}(L) \Phi_{L}\left(v_{L}-v_{12}\right) . \\
& {\left[1-\int_{s}^{s{ }^{2}(X)} n_{1}\left(L^{\prime}\right) k_{12} \Phi_{L^{\prime}}\left(v_{L^{\prime}}-v_{12}\right) \mathrm{d} s^{\prime}\right] \mathrm{d} s \mu^{*} \mathrm{~d} \mu^{*},}
\end{aligned}
$$

$$
X \in\left[X_{\text {med }}^{(2)}, X_{\text {min }}^{(1)}\right],
$$

and where

$$
\begin{aligned}
& \frac{E_{s}^{(2)}(X)}{E_{c}}=\frac{2}{\bar{I}_{c}} \int_{1}^{L_{\text {sup }}(X)} \int_{s p(X)}^{s p(X)} \varepsilon_{12}(L) \Phi_{L}\left(v_{L}-v_{12}\right) . \\
& {\left[1-\int_{s}^{s p^{\prime}(X)} n_{1}\left(L^{\prime}\right) k_{12} \Phi_{L^{\prime}}\left(v_{L^{\prime}}-v_{12}\right) \mathrm{d} s^{\prime}\right] \mathrm{d} s L_{p} \mathrm{~d} L_{p},}
\end{aligned}
$$

if

$$
|X| \leq\left|X_{\min }^{(2)}\right|
$$


The expressions for the frequencies $X_{\text {min }}^{(2)}, X_{\text {med }}^{(1)}$ (which will be later used), $X_{\text {med }}^{(2)}$ and angle cosines $\mu_{1}^{*}, \mu_{2}^{*}$ appearing in the last equation are easily derived on the basis of results (6), (7), and (16)

$$
\begin{aligned}
& X_{\min }^{(2)}=-\sqrt{1-1 / L_{\max }^{2}}-\frac{\Delta v\left(L_{\max }\right) / 2}{v_{\max }-v_{12}}, \\
& X_{\text {med }}^{(1)}=\frac{-v_{0}}{v_{\max }}+\frac{\Delta v(L=1) / 2}{v_{\max }-v_{12}}
\end{aligned}
$$

$v_{0}$ being the radial velocity $v(r)$ at the stellar surface,

$$
\begin{aligned}
& X_{\mathrm{med}}^{(2)}=\frac{\Delta v(L=1) / 2}{v_{\max }-v_{12}}, \\
& \mu_{1}^{*}=\sqrt{1+L_{\max }^{2}\left\{\left(X+\frac{\Delta v\left(L_{\max }\right) / 2}{v_{\max }-v_{12}}\right)^{2}-1\right\}},
\end{aligned}
$$

and

$$
\mu_{2}^{*}=\frac{v_{\max }}{v_{0}}\left(-X+\frac{\Delta v(L=1) / 2}{v_{\max }-v_{12}}\right)
$$

Let us finally note that the upper limit $L_{\text {sup }}(X)$ in Equation (20) depends on both $\Delta v(L)$ and $v(L)$.

\section{First-Order Moment of an Unsaturated P Cygni Profile}

Combining the results obtained in the previous sections with the definition of the first order moment $W_{1} \alpha \int\left(E(\lambda) / E_{c}-1\right)\left(\lambda-\lambda_{12}\right) d \lambda$ of a P Cygni line profile (see CLS and Paper I), we find that

$$
W_{1}=\int_{X_{\min }^{(1)}}^{X_{\max }^{(2)}}\left(\frac{E(X)}{E_{c}}-1\right) X \mathrm{~d} X
$$

Since, in the optically-thin approximation,

$$
\int_{s>s_{1}(X)}^{s{ }^{P}(X)} n_{1}(L) k_{12} \Phi_{L}\left(v_{L}-v_{12}\right) \mathrm{d} s<\tau_{12}^{p}(X)<1,
$$

we may state that the contribution $E_{s}^{(2)}(X) / E_{c}$ to the line profile is an even function of the frequency $X$. Consequently, expression (26) for the first order moment of an 
unsaturated P Cygni profile can easily be reduced to

$$
\begin{aligned}
& W_{1}=\frac{2}{\bar{I}_{c}} \int_{X_{\min }^{(1)}}^{X_{\min }^{(2)}} X \mathrm{~d} X \int_{M a x}^{\operatorname{Inf}\left(1, \mu_{2}^{*}\right)} \mu_{\left.\mu_{1}^{*}\right)}^{*} \mathrm{~d} \mu^{*} \int_{s_{1}^{P}(X)}^{s ?(X)}\left(S_{12}(L)-I_{c} \psi\left(\mu^{*}\right)\right) . \\
& \alpha_{12}(L) \Phi_{L}\left(v_{L}-v_{12}\right) \mathrm{d} s,
\end{aligned}
$$

where

$$
S_{12}(L)=\varepsilon_{12}(L) / \alpha_{12}(L)
$$

represents the source function for the line transition $1 \rightleftharpoons 2$, and

$$
\alpha_{12}(L)=n_{1}(L) k_{12},
$$

the volume absorption coefficient evaluated at a distance $L$ in the rest frame of the fluid.

In a steady state, we find that for a two-level atom model

$$
S_{12}(L)=J_{12}(L)
$$

where, within the optically thin approximation, the mean intensity $J_{12}(L)$ of the radiation field reduces to

$$
J_{12}(L)=I_{c} W^{\psi}(L)
$$

with

$$
W^{\psi}(L)=\frac{1}{2} \int_{0}^{1} \psi\left(\mu^{*}\right) \mu^{*} / L^{2} \sqrt{1-\frac{\left(1-\mu^{* 2}\right)}{L^{2}}} \mathrm{~d} \mu^{*}
$$

being a generalized geometrical dilution factor. Indeed, in the absence of any limb darkening, i.e. assuming $\psi\left(\mu^{*}\right)=1$, we recover the expected result

$$
W(L)=\frac{1}{2}\left(1-\sqrt{1-\frac{1}{L^{2}}}\right) .
$$

In the remainder of this section, we shall reduce Equation (28) to its most simple form. If we visualize first in Figure 3 the domain of integration defined by the first set of integrals in the plane $\left(X, \mu^{*}\right)$, it is straightforward to interchange the order of integration between $X$ and $\mu^{*}$ in Equation (28). Taking into account results (31)-(33), we obtain

$$
\begin{aligned}
W_{1}= & \frac{2 I_{c}}{\bar{I}_{c}} \int_{0}^{1} \mu^{*} \mathrm{~d} \mu^{*} \int_{X_{\mathrm{inf}}^{\mathrm{in}}\left(\mu^{*}\right)}^{X_{\text {sup }}^{(2)}\left(\mu^{*}\right)} X \mathrm{~d} X \int_{s_{1}^{P}(X)}^{s p(X)}\left(W^{\psi}(L)-\psi\left(\mu^{*}\right)\right) \times \\
& \times \alpha_{12}(L) \Phi_{L}\left(v_{L}-v_{12}\right) \mathrm{d} s,
\end{aligned}
$$




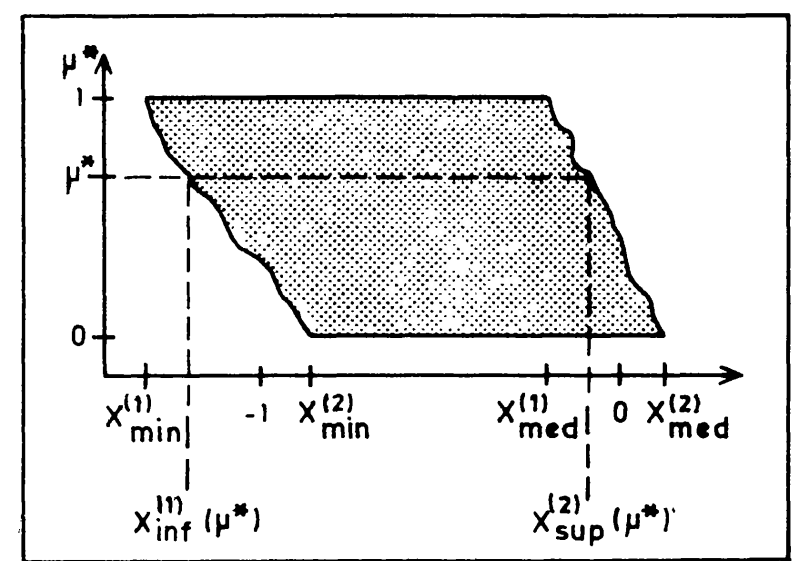

Fig. 3. Domain of integration in the plane $\left(X, \mu^{*}\right)$ for the first pair of integrals in Equation (28). Not drawn to scale.

the expressions of the integration limits $X_{\text {inf }}^{(1)}\left(\mu^{*}\right), X_{\text {sup }}^{(2)}\left(\mu^{*}\right)$ being derived by inverting relations (24) and (25), respectively,

$$
X_{\text {inf }}^{(1)}\left(\mu^{*}\right)=-\sqrt{1-\frac{\left(1-\mu^{* 2}\right)}{L_{\max }^{2}}}-\frac{\Delta v\left(L_{\max }\right) / 2}{v_{\max }-v_{12}},
$$

and

$$
X_{\mathrm{sup}}^{(2)}\left(\mu^{*}\right)=\frac{-v_{0}}{v_{\max }} \mu^{*}+\frac{\Delta v(L=1) / 2}{v_{\max }-v_{12}}
$$

Similarly, after performing in Equation (35) the variable transformation

$$
L=S / \mu, \quad \mathrm{d} L=\mu \mathrm{d} S,
$$

where (see Figure 2)

$$
S=s / R^{*},
$$

and

$$
\mu=\cos (\theta),
$$

and defining the new profile function

$$
\phi_{L}\left(X-X_{L}\right)=\Phi_{L}\left(v_{L}-v_{12}\right),
$$

which is such that

$$
\int_{X_{L}-\left[(\Delta v(L) / 2) /\left(v_{\max }-v_{12}\right)\right]}^{X_{L}+\left[(\Delta v(L) / 2) /\left(v_{\max }-v_{12}\right)\right]} \phi_{L}\left(X-X_{L}\right) \mathrm{d} X=\frac{1}{v_{\max }-v_{12}},
$$




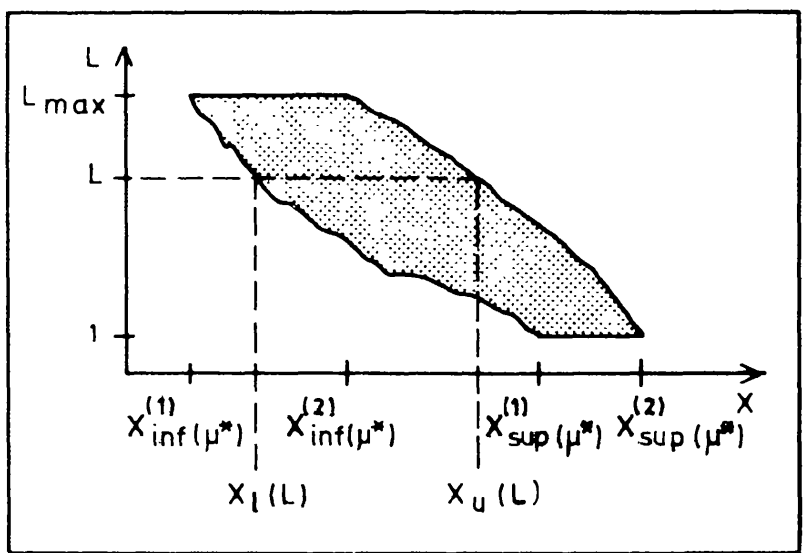

Fig. 4. Domain of integration in the plane $(X, L)$ for the second pair of integrals in Equation (35). Not drawn to scale.

we can easily interchange the order of integration between $X$ and $L$ (see Figure 4 ) in expression (35) to obtain

$$
\begin{aligned}
W_{1}= & \frac{2 I_{c} R^{*}}{\bar{I}_{c}} \int_{0}^{1} \mu^{*} \mathrm{~d} \mu^{*} \int_{1}^{L_{\max }}\left(W^{\psi}(L)-\psi\left(\mu^{*}\right)\right) \frac{\alpha_{12}(L)}{\mu\left(\mu^{*}, L\right)} \mathrm{d} L \times \\
& \times \int_{X_{1}(L)}^{X_{u(L)}} X \phi_{L}\left(X-X_{L}\right) \mathrm{d} X .
\end{aligned}
$$

The lower (1) and upper $(u)$ limits appearing in the last integral are easily derived by setting $v_{L}=v_{12} \pm \Delta v / 2$ in Equation (7)

$$
X_{1}(L)=X_{L}-\frac{\Delta v(L) / 2}{v_{\max }-v_{12}},
$$

and

$$
X_{u}(L)=X_{L}+\frac{\Delta v(L) / 2}{v_{\max }-v_{12}} .
$$

Since we have assumed $\phi_{L}\left(X-X_{L}\right)$ to be a symmetric function and taking into account results (6) and (41), it is straightforward to perform the last integration in Equation (42). We readily obtain

$$
\begin{aligned}
W_{1}= & -\frac{2 I_{c} R^{*}}{\bar{I}_{c}} \int_{0}^{1} \mu^{*} \mathrm{~d} \mu^{*} \int_{1}^{L_{\max }}\left(W^{\psi}(L)-\psi\left(\mu^{*}\right)\right) \times \\
& \times \alpha_{12}(L) \frac{v(L)}{v_{\max }} \frac{\mathrm{d} L}{\left(v_{\max }-v_{12}\right)} .
\end{aligned}
$$


Making use of relations (3), (8), (18), (30) and expressing the ground level population $n_{1}(L)$ in terms of the mass-loss rate $\dot{M}$ of the central object (see CLS), Equation (44) may be rewritten in a more elegant form as

$$
\begin{aligned}
W_{1}= & \frac{\pi e^{2}}{m c} f_{12} \lambda_{12} \frac{A(\text { element }) \dot{M}}{4 \pi \bar{\mu} M_{\mathrm{amu}} v_{\max }^{2} R^{*}} \int_{1}^{L_{\max }}\left(\frac{I_{c}}{\bar{I}_{c}} W^{\psi}(L)-1\right) \times \\
& \times \frac{n(\text { level })}{L^{2}} \mathrm{~d} L,
\end{aligned}
$$

where $n$ (level) represents the fractional abundance of the relevant ion in the ground state, $A$ (element) the abundance of the element itself, $\bar{\mu}$ the mean atomic weight of the nuclei in the flow, $M_{\mathrm{amu}}$ the unit of atomic mass and $\lambda_{12}$ the wavelength of the line transition $1 \rightleftharpoons 2$.

Referring the reader to Equations (60) and (74)-(78) of Paper I, we can directly state that expression (45) derived here for the first order moment $W_{1}$ of an unsaturated P Cygni line profile is identical to that obtained in the pure formalism of Sobolev-type approximations used for the transfer of line radiation. The meaning of this conclusion namely implies that all results derived in Paper I, and concerning mainly the nondependence of $W_{1}$ - and subsequently the derivation of $\dot{M}$ - vs various physical and geometrical effects, equally apply for the case of an arbitrary (e.g. non-Sobolev type) outward-accelerating envelope. As an example, we have demonstrated in Paper I that in the context of 'very rapidly' expanding envelopes, neglecting a strong limb darkening effect for the central source in the expression of $W_{1}$ would lead to an overestimate of the mass-loss rate $\dot{M}$ by a factor as small as $1 \%$. Consequently, within a very good approximation, we may replace Equation (45) by

$$
W_{1}=\frac{\pi e^{2}}{m c} f_{12} \lambda_{12} \frac{A(\text { element }) \dot{M}}{4 \pi \bar{\mu} M_{\mathrm{amu}} v_{\max }^{2} R^{*}} \int_{1}^{L_{\max }}(W(L)-1) \frac{n(\text { level })}{L^{2}} \mathrm{~d} L,
$$

where the geometrical dilution factor $W(L)$ has been defined in (34).

Assuming now that the fractional abundance $n$ (level) is a constant across the medium, i.e. there is mass conservation of the species under study, the expression of the first order moment $W_{1}$ takes the final form

$$
W_{1}=\frac{\pi e^{2}}{m c} f_{12} \lambda_{12} \frac{A(\text { element }) \dot{M} n(\text { level })}{4 \pi \bar{\mu} M_{\mathrm{amu}} v_{\max }^{2} R^{*}} q^{c}\left(L_{\mathrm{max}}\right)
$$

with

$$
\begin{aligned}
q^{c}\left(L_{\max }\right)= & -\left(\frac{1}{2}+\frac{\pi}{8}\right)+\frac{1}{2 L_{\max }}+\frac{1}{4 L_{\max }} \sqrt{1-\left(\frac{1}{L_{\max }}\right)^{2}}+ \\
& +\frac{1}{4} \arcsin \left(\frac{1}{L_{\max }}\right) .
\end{aligned}
$$


Although the approximation of mass conservation should be essentially good for resonance lines of dominant ions in the flow, it could be inappropriate for some other ions. If this is the case, we may overcome the difficulty by replacing $n$ (level) in Equation (47) by

$$
\bar{n}(\text { level })=\int_{1}^{L_{\max }}(W(L)-1) \frac{n(\text { level })}{L^{2}} \mathrm{~d} L / \int_{1}^{L_{\max }}(W(L)-1) \frac{\mathrm{d} L}{L^{2}},
$$

i.e. a representative value for the fractional abundance of the relevant ion in the flow.

Finally, regarding the dependence of $W_{1}-$ or $\dot{M}$ - onto the 'a priori' unknown value $L_{\max }$ for the size of the expanding envelope, we have already established in Paper I that replacing $q^{c}\left(L_{\max }\right)$ by its asymptotic expression $q^{c}(\infty)=-0.8927$ in Equation (47) will just result in underestimating the mass-loss rate $\dot{M}$ by a factor never exceeding $30 \%$.

\section{Conclusions}

Irrespective of any Sobolev-type approximations used for the transfer of line radiation in outward-accelerating envelopes, we have demonstrated in the present work that a unique relation does actually exist between the mass-loss rate $\dot{M}$ of a central star and the first order moment $W_{1}$ of an unsaturated $\mathrm{P}$ Cygni profile. On speculative physical grounds, this result is not so surprising since the optically thin approximation enables one to avoid, strictly speaking, the true problem of radiative transfer... it is as if at different frequencies, the observer were seeing at different depths the cross-section of each individual ion in front of the stellar disk, thus measuring $W_{1}$, or similarly $\dot{M}$.

Adopting cosmic abundances (Allen, 1976), we derive from Equation (47) the very useful relation

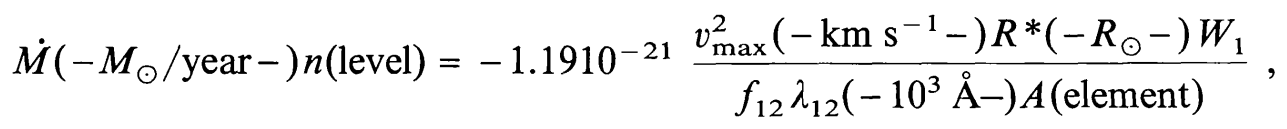

where, as usually, $M_{\odot}$ and $R_{\odot}$ stand for the solar mass and radius units. Since this relation is identical to that derived in Paper I for the case of 'very rapidly' expanding atmospheres and that we have already investigated there its dependence vs various physical and geometrical effects, we summarize and generalize hereafter the main conclusions of that study:

- With $n$ (level) being a representative value (cf. Equation (49)) for the fractional abundance of the relevant ion in the flow, Equation (50) allows a direct determination of the mass-loss rate $\dot{M}$, irrespective of the type of radial velocity field $v(r)$ characterizing the expansion of the envelope.

- Considering the effects due to collisions $(\varepsilon \neq 0)$ and/or the superposition of a rotational velocity field $v^{\perp}(r)$ in the atmosphere, we can state that none of these effects modifies relation $(50)$. 
- Neglecting the presence of an underlying photospheric absorption line has the net result of underestimating the mass-loss rate by a factor of $20 \%$ at maximum.

- For the case of an unresolved resonance multiplet profile, care should be taken in adopting the wavelength $\lambda_{D}$ and the total oscillator strength $f_{D}$ of the multiplet when calculating $W_{1}$ (see Equation (7) of Paper I) and $\dot{M}$ from Equation (50).

- Due to the fact that we have implicitly used the asymptotic value $q^{c}(\infty)$ instead of $q^{c}\left(L_{\max }\right)$ (see Equation (48)) when deriving relation (50), there results a possible underestimate of the mass-loss rate by a factor $\leq 30 \%$.

- As to the limb darkening, its effects are always negligible.

Finally, referring the reader to the work by CLS for the possible error $(\sim 10 \%)$ affecting the measurement of $W_{1}$ from an observed line profile, we may safely conclude that, within a total uncertainty less than roughly $60 \%(20 \%+30 \%+10 \%)$, Equation (50) offers a powerful method for deriving mass-loss rates from the first order moment of unsaturated P Cygni profiles. Let us still stress that this method can be applied - indistinctly - to any of the early- as well as late-type stars, quasars, etc. known to be losing mass.

\section{Acknowledgement}

It is a pleasure to thank Jean-Pierre Swings for critical reading of the manuscript.

\section{References}

Allen, C. W.: 1976, Astrophysical Quantities, 3rd edition, University of London, The Athlone Press.

Castor, J. I.: 1970, Monthly Notices Roy. Astron. Soc. 149, 111.

Castor, J. I., Lutz, J. H., and Seaton, M. J.: 1981, Monthly Notices Roy. Astron. Soc. 194, 547 (CLS).

Lucy, L. B.: 1971, Astrophys. J. 163, 95.

Sobolev, V. V.: 1947, Dnižuščiesja Oboločki Zvězd, Leningrad (Moving Envelopes of Stars, translated from Russian by S. Gaposchkin, Harvard University Press, Cambridge, Mass., 1960).

Sobolev, V. V.: 1957, Astron. Z. 34, 694 (translated Soviet Astron. 1, 678).

Sobolev, V. V.: 1958, in V. A. Ambartsumyan (ed.), Theoretical Astrophysics, Chapter 28, Pergamon Press Ltd., London.

Surdej, J.: 1982, Astrophys. Space Sci. 88, 31. 